\title{
Substrate Independent Microwave Components in Substrate Integrated Waveguide Technology for High-Performance Smart Surfaces
}

\author{
Kamil Yavuz Kapusuz, Student Member, IEEE, Sam Lemey, Member, IEEE, \\ and Hendrik Rogier, Senior Member, IEEE
}

\begin{abstract}
Although all existing air-filled substrate integrated waveguide (AFSIW) topologies yield substrate-independent electrical performance, they rely on dedicated, expensive, laminates to form air-filled regions that contain the electromagnetic fields. This paper proposes a novel substrate-independent AFSIW manufacturing technology, enabling straightforward integration of highperformance microwave components into a wide range of generalpurpose commercially-available surface materials by means of standard additive (3D printing) or subtractive (computer numerically controlled milling/laser cutting) manufacturing processes. First, an analytical formula is derived for the effective permittivity and loss tangent of the AFSIW waveguide. This allows the designer to reduce substrate losses to levels typically encountered in high-frequency laminates. Then, several microwave components are designed and fabricated. Measurements of multiple AFSIW waveguides and a four-way power divider/combiner, both relying on a new coaxial-to-air-filled SIW transition, prove that this novel approach yields microwave components suitable for direct integration into everyday surfaces, with low insertion loss, and excellent matching and isolation over the entire $[5.15-5.85] \mathrm{GHz}$ band. Hence, this innovative approach paves the way for a new generation of cost-effective, high-performance and invisibly-integrated smart surface systems that efficiently exploit the area and the materials available in everyday objects.
\end{abstract}

Index Terms-Additive manufacturing, AFSIW transition, airfilled substrate integrated waveguide (AFSIW), loss reduction, power divider, smart surface, subtractive manufacturing.

\section{INTRODUCTION}

$\mathbf{T}$ HE Internet of Things (IoT) envisages a worldwide infrastructure of interconnected everyday objects, augmented with additional sensing, computing and wireless communication functionality, thereby enabling intelligent and independent interaction between objects and their surroundings [1]. To unleash the full potential of the IoT paradigm, there is a stringent need for low cost and low profile microwave systems that facilitate invisible integration into common surfaces such as floors, walls, textile sheets and worktops, without significantly altering their size, weight, cost, aesthetics or original purpose [2]. In addition, such microwave smart surface systems should provide high performance (good matching, low loss and high isolation) over

Manuscript received September 29, 2017; revised December 22, 2017; accepted March 15, 2018. Date of publication ; date of current version . This work was supported in part by the European Research Council grant 695495 "ATTO: A new concept for ultra-high capacity wireless networks".

K.Y. Kapusuz, S. Lemey and H. Rogier are with the IDLabElectromagnetics Group, Department of Information Technology, Ghent University-IMEC, Technologiepark 15, Ghent B-9052, Belgium. (e-mail: kamilyavuz.kapusuz@ugent.be; sam.lemey@ugent.be; hendrik.rogier@ugent.be). a wide frequency band to support the ever-increasing volumes of data [3] and to guarantee their performance when integrated into a wide variety of common objects and under realistic, timevarying operating conditions. Thereby, numerous application domains will benefit from smart surface technology, such as smart-fabric interactive-textiles [4], smart furniture [5] and smart domotics [6].

Substrate Integrated Waveguide (SIW) technology and, more generally, Substrate Integrated Circuit (SIC) architecture, have already shown great potential for unobtrusive and robust integration of low-profile planar microwave circuits into common surfaces [7], [8]. By confining the electromagnetic fields [9], their waveguide topology yields high isolation and suppresses surface waves. Moreover, various bandwidth enhancement techniques enable designers to meet the demanding requirements of the current and next generation IoT applications [10]. Furthermore, SIC microwave components and systems may be implemented with innovative materials through various manufacturing techniques [11]. Interesting for the IoT are the opportunities of exploiting the materials available in everyday objects to realize the SIC structure, as this yields a significant cost and area reduction [12]. However, this requires materials with appropriate RF characteristics, such as low moisture regain, low losses, and consistent batch-to-batch characteristics. In conventional dielectric-filled SIC architectures, the electromagnetic characteristics of a dielectric substrate will affect performance in a frequency selective manner and cause losses. Therefore, in the last decade, air-filled SIW (AFSIW) was introduced to reduce the dielectric loss and to improve the power handling capability of the SIC topology by replacing the dielectric substrate material by air [13]. A wide variety of low-loss AFSIW designs have been proposed in literature, such as dielectric-to-air-filled SIW transitions [14], filters [15], and phase shifters [16].

This paper introduces an innovative AFSIW manufacturing technology for invisible and robust integration of highperformance microwave components inside a wide variety of traditional everyday surfaces, through standard additive or subtractive manufacturing processes. This novel approach yields: (i) high performance, independent of the adopted surface material (ii) compatibility with standard subtractive and additive manufacturing processes, (iii) arbitrary selection of the substrate thickness, (iv) direct, easy and invisible integration inside traditional, everyday lossy surfaces, (v) low material and production costs, (vi) stable performance after surface integration, and 
(vii) a straightforward design procedure based on a novel analytical formula for the effective permittivity and loss tangent of AFSIW waveguides. To the authors' best knowledge, this is the first contribution that exploits the opportunities of traditional everyday surface materials to realize low-cost and wideband AFSIW systems. Several design examples illustrate and validate the advantages of this novel technology. First, a coaxial-to-airfilled SIW transition is presented, which is then applied in the design of compact, cost-effective, low-profile waveguides and of a novel four-way power divider/combiner. All components were optimized for operation in the $5.15 \mathrm{GHz}$ to $5.85 \mathrm{GHz}$ frequency band, which contains all unlicensed national information infrastructure (U-NII) radio bands. This makes these components suitable for use in Wi-Fi access points, such as $802.11 \mathrm{ac}$ stations. The performance of these components is validated after implementation in three general-purpose surface materials through standard additive and subtractive manufacturing processes.

Over the last few years, diverse fabrication methodologies [17]-[23] have been developed to manufacture AFSIW components. [17] proposes a special high-resolution micromachining-based technique for application at millimeter-wave and terahertz frequencies [17]. The approach in [18] is based on low temperature co-fired ceramics (LTCC) technology. Although both technologies are promising high performance and high frequency solutions, they are expensive and, therefore, not ideal to manufacture low-cost large-area products in the lower microwave frequency range. [19]-[23] rely on standard PCB processing, including substrate milling and metallization procedures, to implement cost-effective AFSIW systems in commercially available PCB substrates. [19] realize AFSIW walls through pin plating in milled-out high-frequency PCB material. [20] applies an additional glue layer and edge plating to form AFSIW structures. [21]-[23] present another multilayer AFSIW manufacturing technology: to realize the sidewalls, the inner borders of the milled-out PCB substrate are metallized using edge-plating without any additional glue layer. Even though these solutions yield a lower fabrication cost and easier assembly of multilayer AFSIW structures, they are restricted to those materials that comply with the requirements of edge metallization processes, thereby ruling out most of the traditional everyday surface materials. Moreover, edgeplating-based SIW topologies [21]-[23] may require stacking multiple edge-plated PCBs to realize thick AFSIW structures. In contrast, our novel approach complies with a wide variety of traditional everyday surfaces and the thickness of the SIC can be arbitrarily selected without requiring additional PCBs

In the remainder of the paper, Section II outlines the surfacematerial-based AFSIW technology and IoT integration strategy, including the fabrication procedures for different surface materials and manufacturing processes. Section III analyses the critical design parameters, including propagation factor and total transmission loss. In Section IV, several design examples are realized and validated to demonstrate the huge potential of this novel manufacturing and integration technology. Finally, conclusions are drawn in Section V.

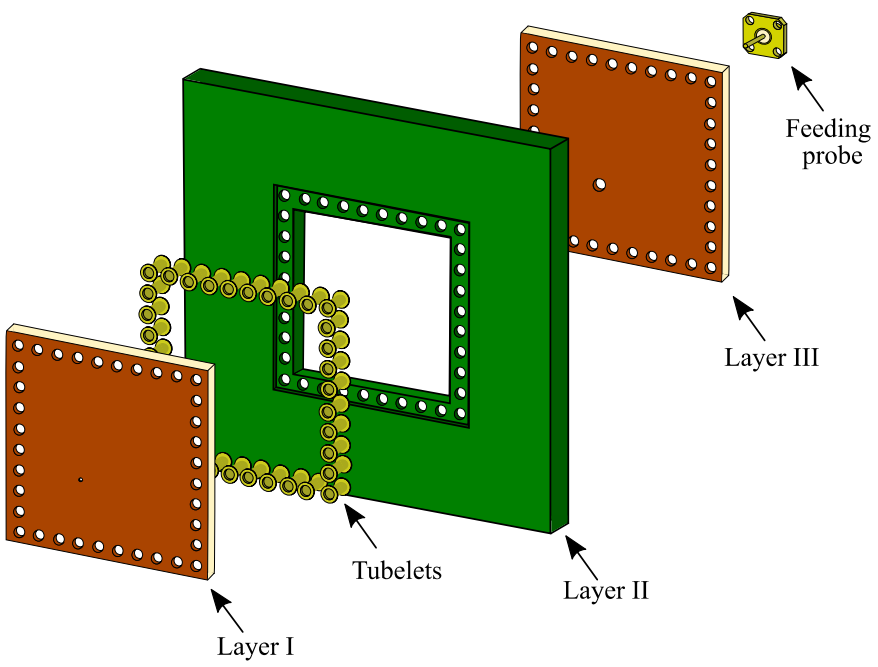

(a)

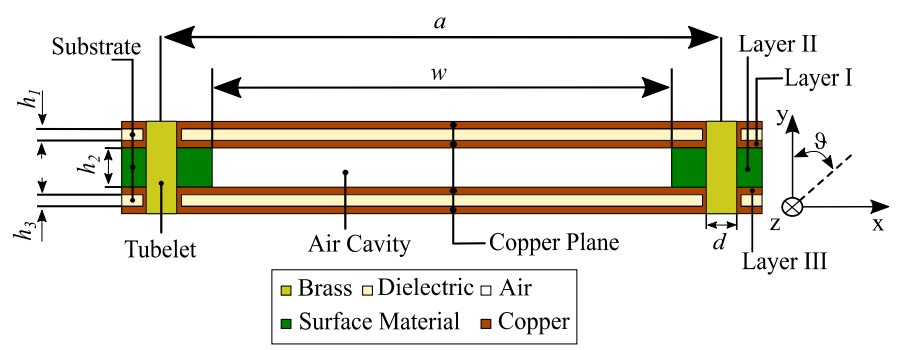

(b)

Fig. 1. Concept and architecture of the surface-material-based AFSIW technology. (a) Exploded view. (b) Cross-sectional view.

\section{Substrate-IndePendent SIW TeChnology FOR SMART SURFACE SYSTEMS}

This section introduces our novel AFSIW technology. It describes both subtractive and additive cost-effective manufacturing processes to robustly and invisibly implement highperformance microwave components in traditional everyday surfaces, such as furniture, casings, floor and worktops.

\section{A. Concept and Architecture}

The next generation high-performance smart surface systems require invisibly-integrated, low-cost, low-profile microwave components. To keep both cost and size to the absolute minimum, all microwave components may be directly implemented in the surface material by conventional dielectric-filled SIW technology [6], [8]. However, adopting commonly-used surface materials as dielectric substrates may raise various issues [12], [24]. Typically, general-purpose off-the-shelf large-surface materials do not provide the required electromagnetic microwavefrequency substrate performance, due to their inhomogeneity and high losses. Furthermore, their permittivity and loss tangent are frequency dependent, vary from batch to batch and differ from surface to surface (for example, wood versus cork).

A new AFSIW manufacturing technology is proposed to obtain high-performance substrate-independent microwave components. Fig. 1 depicts the concept and architecture. The structure is composed of two conductive layers (Layer I and III), one layer of general purpose off-the-shelf surface material (Layer II) 
and low-cost conductive tubelets. Substrate-independent behavior is then obtained by patterning in Layer II a well-defined air cavity, serving as transmission medium for the electromagnetic fields. It is created by either removing parts of the off-theshelf surface material or realizing the general-purpose surface material through additive manufacturing. Hence, our approach minimizes the electromagnetic fields in the lossy off-the-shelf surface material, without requiring costly high-frequency laminates. To avoid that electromagnetic fields penetrate the lossy surface material, sidewalls are implemented by creating via holes in the surface material, at locations that are very close to the edges of the air cavity. These holes are then metallized by punching conductive tubelets through them, thereby joining all layers [Fig. 1(a)]. A wide range of microwave components are obtained by judiciously patterning these low-cost brass tubelet sidewalls and the air cavity. Fig. 1 shows that a small portion of the dielectric material (Layer II) remains inside the air cavity for mechanical integrity. Yet, the lower-order modes in a waveguide or a cavity have their highest electric field sufficiently far away from the cavity sidewalls. Near the edge, where a small portion of lossy dielectric remains present, serving as support for the tubelets, the field values remain small. Therefore, those remaining dielectric slabs only introduce a very small loss. In Section IV-A, we show that, for ratios of $w / a$ above 0.9 , this loss is almost independent of the dielectric properties of Layer II for the lower-order modes. In that case, any dielectric surface material can be applied as Layer II.

\section{B. Subtractive Manufacturing}

A first way to shape Layer II [Fig. 1] consists in milling/cutting out the desired shape in a commercially available wood- or plastic-based panel material by means of computer numerically controlled (CNC) milling or laser cutting, both being conventional fabrication processes. They are commonly used to manufacture furniture (desks, closets, tables, ... ) and/or laminate floor tiles. The process implements both the air cavity, of width $w$ [Fig. 1(b)], and the cylindrical holes with diameter $d$. The BRM100160 laser cutter, applied in this paper, is able to create cavities of arbitrary shape in a volume of $1000 \times 1600 \times 230 \mathrm{~mm}^{3}$, with an accuracy of $0.1 \mathrm{~mm}$.

\section{Constructive (Additive) Manufacturing}

As an alternative, the dielectric material of Layer II may be $3 \mathrm{D}$ printed, without requiring any removal of material, through fused deposition modelling (FDM), polyjet, stereo-lithographic apparatus (SLA), selective laser sintering (SLS) and selective laser melting (SLM). These methods yield different surface roughness resolutions, at different manufacturing costs [25]. In this paper, the FDM method by Ultimaker 2 is adopted, given its lowest manufacturing cost and decent surface roughness [26]. Yet, it only allows printing plastics. The machine is able to print a volume of $342 \times 357 \times 388 \mathrm{~mm}^{3}$ with vertical resolution of $5 \mu \mathrm{m}$ and with azimuthal resolution of $12.5 \mu \mathrm{m}$. On the one hand, the extrusion speed and temperature, layer thickness, infill pattern and percentage are important parameters to achieve the desired electromagnetic properties and to obtain a reproducible manufacturing process. Hence, a proper combination of this large number of options needs to be carefully selected for dielectric-filled microwave structures. On the other hand, our novel AFSIW topology yields microwave components for smart surfaces with properties independent of the substrate's dielectric characteristics, irrespective of the fabrication settings. Therefore, small changes in the 3D-printing process, such as extrusion speed and temperature, infill pattern and percentage, do not affect performance of such components. Hence, by implementing Layer II by means of a standard 3D-printing technique, its main advantages [27] are exploited, being the ability to implement arbitrarily shaped planar SIW structures with arbitrary thickness in a cost-effective manner, while avoiding its disadvantages, being a performance that strongly depends on material and process parameters, by inserting well-defined air-filled regions [27].

\section{Assembly of Layers}

We now describe the three-step assembly process to invisibly integrate our AFSIW microwave components into smart surfaces. First, an off-the-shelf surface material (Layer II) is shaped such that the highest electric fields occur in air-filled regions and that holes are implemented for the insertion of commercial low-cost tubelets. Depending on the selected surface material, Layer II is produced through either subtractive (Section II.B) or additive (Section II.C) manufacturing. Second, the structure is closed by inserting tubelets through the alignment holes to fix conductive layers I and III on both sides of Layer II. In this paper, low-cost metallized PCB substrates, such as FR4, are used to implement Layer I and Layer III. Yet, any nonmagnetic conductive sheet may serve as alternative. Third, the complete assembly is joined by punching these brass tubelets, thereby also forming the sidewalls of the AFSIW components. They are spaced closely enough to minimize radiation losses. The length of the tubelets, together with the thickness of Layer II $\left(h_{2}\right)$, determines the height of the SIW sidewalls. Thereby, thick AFSIW cavities can be easily constructed by using thicker surface material with longer tubelets. In contrast, in a standard PCB manufacturing process, thicker substrates $\left(h_{1}+h_{2}+h_{3}>2.5 \mathrm{~mm}\right)$ are not preferred for manufacturing SIW components, due to via-hole metallization issues [10]. Moreover, the use of tubelets preserves the flexibility of conformal microwave component designs [28]. For quick prototyping purposes, the tubelets are fixed at their predefined positions by a manual tubelet-punching machine. When moving to industrial mass production, fully-automated tubelet machines or $\mathrm{CNC}$ tubelet-punching machines could be used.

Hence, the above approach yields high-performance microwave components in commercially-available general-purpose low-cost surface materials. In contrast to state-of-the-art AFSIW technology, our manufacturing process provides unprecedented freedom in selecting substrate materials, while CNC lasercutting/milling or 3D printing enables a wide range of cavity thicknesses $\left(h_{2}\right)$. Furthermore, the direct and invisible integration of such microwave components in the surface material significantly reduces production and integration costs of smart surface systems, since the readily available material and volume are efficiently reused to implement additional functionality. Finally, in contrast to pin plating in standard PCB processing, there is no need to put dielectric slabs to support inductive posts, 


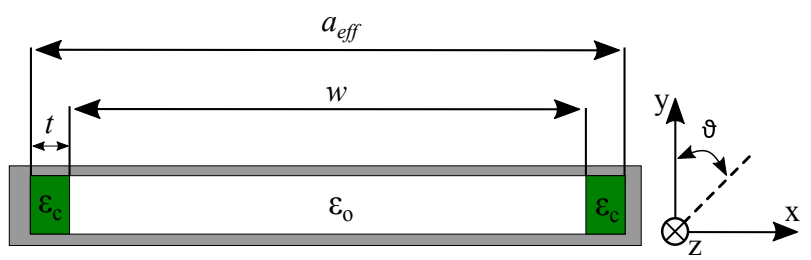

(a)

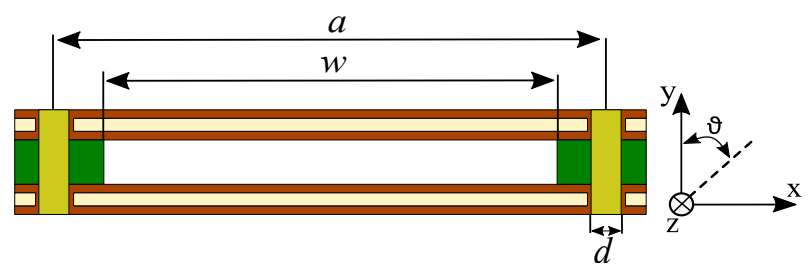

(b)

Fig. 2. (a) Cross-sectional view of a double dielectric-slab-loaded rectangular waveguide, (b) Cross-sectional view of an AFSIW.

such as in the design of air-filled hybrid couplers [14], power dividers [14], and filters [15].

\section{Performance Analysis}

\section{A. Propagation Constant and Effective Complex Permittivity}

To theoretically analyze performance, we consider a waveguide implemented in the AFSIW manufacturing technology described above. Fig. 2(b) depicts its cross-section, while Fig. 2(a) shows the cross-section of an equivalent double dielectric-slabloaded rectangular waveguide. It is well-known that such structures support longitudinal section electric and magnetic modes ( $L S E$ and $L S M$ modes), having the electric and magnetic vector contained entirely within a longitudinal section [29], rather than pure transverse electric and magnetic modes ( $T E$ and $T M$ modes). Given that the rows of vias only support yoriented currents, only $L S E_{n 0}$ modes can propagate within the AFSIW. For mode propagation along the $z$-direction according to an exponential factor $e^{-\gamma z}$, the propagation constants $\gamma$ satisfy [29]

$$
\gamma^{2}=l^{2}-k_{0}^{2}=h^{2}-\epsilon_{c} k_{0}^{2} .
$$

where $h$ and $l$ are the transverse wavenumbers in dielectric and air, $k_{0}=2 \pi / \lambda_{0}$ is the wavenumber, $\lambda_{0}$ is the free-space wavelength, and $\epsilon_{c}$ is the permittivity of the dielectric slabs inside the waveguide (i.e. permittivity of the surface material). The $L S E_{n 0}$ modes' propagation constants $\gamma$ are then found by solving

$$
\begin{aligned}
& h \cot h t=l \tan \frac{l w}{2} \\
& l \tan h t=-h \tan \frac{l w}{2}
\end{aligned}
$$

for odd and even mode numbers $n$, respectively. The effective thickness $t$ of the lossy material serving as sidewall support may be approximated by [30]

$$
t=\frac{a-w}{2}-s\left(0.383 e^{0.4482 d / s}-0.588 e^{-1.214 d / s}\right)
$$

where $d$ is the diameter of the vias forming the sidewalls and $s$ is the spacing between them. Note that other analytical approximations for $t$ may be used instead without affecting the theory below.

We now consider the case where the effective thickness $t$ is small with respect to the total waveguide width $a$. We may then apply a perturbation analysis by substituting the approximation

$$
l \approx \frac{n \pi}{w}+\alpha_{1} t+\alpha_{2} t^{2}+\alpha_{3} t^{3}+\alpha_{4} t^{4}, \quad \text { for } t \ll w,
$$

in (2) and (1), for odd $n$, and in (3) and (1), for even $n$. Both procedures yield:

$$
\begin{aligned}
\alpha_{1} & =-\frac{2 n \pi}{w^{2}} \\
\alpha_{2} & =\frac{4 n \pi}{w^{3}} \\
\alpha_{3} & =-\frac{24 n \pi}{3 w^{4}}-k_{0}^{2}\left(\epsilon_{c}-1\right) \frac{2 n \pi}{3 w^{2}} \\
\alpha_{4} & =\frac{48 n \pi}{3 w^{5}}+k_{0}^{2}\left(\epsilon_{c}-1\right) \frac{8 n \pi}{3 w^{3}}
\end{aligned}
$$

Notice that the contrast in dielectric constant due to the lossy material supporting the sidewalls only starts to affect the propagation from the term in $t^{3}$. By relying on this observation, and by inserting the Taylor expansion for $l^{2}$ into (1) and rearranging the terms, we finally obtain as an approximation for small relative effective thicknesses $t / w$ of the supporting sidewalls

$$
\gamma^{2} \approx\left(\frac{n \pi}{w+2 t}\right)^{2}-k_{0}^{2}\left[1+\left(\epsilon_{c}-1\right)(n \pi)^{2}\left(\frac{4 t^{3}}{3 w^{3}}-\frac{8 t^{4}}{w^{4}}\right)\right] .
$$

We conclude that, for small relative effective thicknesses $t / w$ of the supporting sidewalls, the proposed AFSIW behaves as a homogeneous waveguide with an effective complex permittivity

$$
\epsilon_{c, \text { eff }} \approx 1+\left(\epsilon_{c}-1\right)(n \pi)^{2}\left(\frac{4 t^{3}}{3 w^{3}}-\frac{8 t^{4}}{w^{4}}\right)
$$

depending on terms of third and higher orders in $t / w$. It is intuitively clear that the propagation constant of higher-order modes is more affected by the lossy material than that of the fundamental mode.

\section{B. Loss Mechanisms}

Besides the dielectric losses due to the substrate material supporting the side walls, radiation losses, due the leakage through the gaps between tubelets, and conductor losses in the metal will also be present. Hence, the total attenuation, $\alpha_{t}$, $(\mathrm{Np} / \mathrm{m})$ is given by

$$
\alpha_{t}=\alpha_{d}+\alpha_{r}+\alpha_{c} * K
$$

where $\alpha_{d}$ is the dielectric loss attenuation, $\alpha_{r}$ is the radiation loss attenuation, $\alpha_{c}$ is the conductor loss attenuation, and $K$ is the surface roughness coefficient. Minimal total attenuation can only be obtained when each individual loss term is minimized.

Following (11), the attenuation constant, $\alpha_{d}$, can be made small by minimizing the $t / w$ ratio. However, a realistic AFSIW requires that small dielectric slabs remain inside the waveguide to guarantee sufficient mechanical strength. The initial design procedure, outlined in Section IV-A, leverages (11) to determine the optimal $t / w$ that yields an acceptably small $\alpha_{d}$. 
Radiation loss attenuation, $\alpha_{r}$, is due to leakage through the gaps between SIW walls. Leakage loss is minimized by applying low-cost brass tubelets that are spaced closely enough to minimize leakage loss $(1.6<s / d<3.0$, and $\lambda_{c} / 20<s<\lambda_{c} / 4$, where $s$ is the distance between the center of the tubelets, $d$ is the diameter of the tubelets and $\lambda_{c}$ is the cutoff frequency of the waveguide) [31]. Electromagnetic field simulations have shown that the step in dielectric contrast in this AFSIW configuration only has a marginal effect on its radiation losses and that, therefore, design rules for homogeneous SIW waveguides may be applied in terms of leakage loss.

Conductor loss attenuation, $\alpha_{c}$, is due to the finite conductivity of AFSIW walls. It becomes the main contributor in AFSIW after reducing the dielectric and radiation losses. For standard PCB manufacturing techniques, [14] demonstrated that the surface roughness of the outer layer of the copper cladding, located at the conductor/air-cavity interface, is substantially smaller than the surface roughness of the inner layer of the cladding, at the conductor/substrate interface. In contrast to conventional dielectric-filled SIW structures, where the inner cladding layers surface roughness needs to be taken into account, for AFSIW structures the outer surface roughness of the copper foil needs to be considered. Moreover, surface roughness loss of the AFSIW can be reduced further by smoothing the outer surface of the copper claddings of layers I and III before assembly. Hence, unlike the dielectric-filled SIW, the conductivity value of the surface can be further improved by applying an appropriate plating process with a high conductivity material. However, while our novel manufacturing technology opens new perspectives to exploit a wide variety of surface finishing techniques to further enhance performance, a standard low-cost PCB surface finishing technique is more than sufficient for adequate performance in the U-NII radio communication band. Hence, unlike the dielectric-filled SIW, the conductivity value of the surface can be further improved by applying an appropriate plating process with a high conductivity material. Furthermore, AFSIW yields lower conductor loss since the intrinsic impedance of the dielectric-filled SIW is smaller than that of the AFSIW [32]. In addition, our novel manufacturing technology enables the implementation of AFSIW structures with arbitrary thickness, thereby enabling designers to further reduce conductor loss [31].

\section{DESIGN EXAMPLES FOR SMART SURfACE INTEGRATION}

To validate our novel AFSIW manufacturing technology and to demonstrate its potential, various microwave components have been designed, fabricated and analyzed in three commonly used off-the-shelf surface materials by means of a standard additive and subtractive manufacturing process. Therefore, in the initial design procedure, we first characterize the electromagnetic properties of the surface materials, adopted in different manufacturing technologies, such as standard CNC milling, lasercutting and 3D printing. Following the theory of Section III-A, we then fix the thickness of the lossy sidewall supports to guarantee acceptably small levels of effective dielectric contrast and loss tangent. After these steps, the actual design of microwave components in the proposed technology starts.
First, a substrate independent wideband coaxial-air-filled SIW transition is discussed. Next, the transition is applied in the implementation of compact, cost-effective, low-profile waveguides and of a novel four-way power divider/combiner. All microwave components operate within the entire frequency band from $5.15 \mathrm{GHz}$ to $5.85 \mathrm{GHz}$, thereby covering all unlicensed national information infrastructure (U-NII) radio bands. Hence, they are suitable for use in Wi-Fi access points, such as 802.11ac stations.

\section{A. Initial Design Procedure}

The initial design procedure ensures high performance of the designed components with figures of merit that are independent of the off-the-shelf substrate and the manufacturing process. Therefore, we first characterize the different surface materials under consideration and fix the thickness of the sidewall supports implemented in these materials. In this paper, mediumdensity fibreboard (MDF), polymethyl methacrylate (PMMA, Plexiglass) and polylactic acid (PLA), a 3D printing material, are chosen as sample surface materials. MDF is one of the most commonly used materials to manufacture furniture, such as desks, closets and tables. PMMA is a commonly used shatterresistant alternative for glass, whereas PLA is one of the most common and cost-effective solutions for 3D-printing. As these off-the-shelf surface materials are not originally intended for design of microwave components, their dielectric properties cannot be easily extracted from datasheets. Therefore, we apply a matrix-pencil two-line method [33] to characterize the electrical characteristics of the selected sample materials.

The MDF and PMMA substrates are manufactured by standard laser cutting, as explained in Section II.B, while three PLA substrates are 3D printed, following Section II.C. The MDF and PMMA substrates may also be realized through standard milling (Section II.B). The dielectric constant and loss tangent of MDF are characterized to be 2.21 and 0.06 over the entire U-NII radio communication band from $5.15 \mathrm{GHz}$ to $5.85 \mathrm{GHz}$, whereas the measured dielectric constant and loss tangent of PMMA equal 2.50 and 0.094 , respectively.

The substrates of 3D printed materials with infill factors of $25 \%, 50 \%$, and $100 \%$ exhibit a dielectric constant of 1.65 , $2.0,3.05$ and a loss tangent of $0.015,0.03,0.05$, respectively. Note that all values specified above refer to the nominal dielectric properties of the material. For all materials under study, the dielectric properties are frequency dependent and vary from batch to batch due to inhomogeneity caused by their cellular structure. Moreover, both the infill percentage and small variations in extrusion speed and temperature during the 3Dprinting process affect the dielectric properties of the $3 \mathrm{D}$-printed material [34].

Yet, these estimated material characteristics only serve to obtain estimates of the effective dielectric constant and effective loss tangent of an AFSIW waveguide, based on (11). First, we validate the formula for a waveguide with MDF supporting walls. The waveguide width equals $w=0.65 \lambda_{0}$ [Fig. 2(b)], with $\lambda_{0}$ the free-space wavelength. Fig. 3 depicts the exact effective loss tangent as a function of the effective thickness $t / \lambda_{0}$ of the supporting side walls, along with the third-order and fourth-order approximations. Both the fundamental propagation 


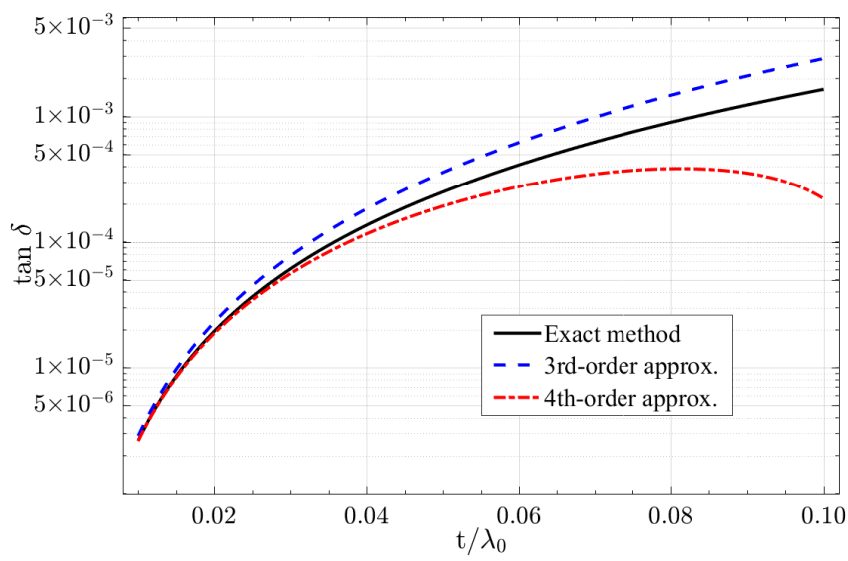

(a)

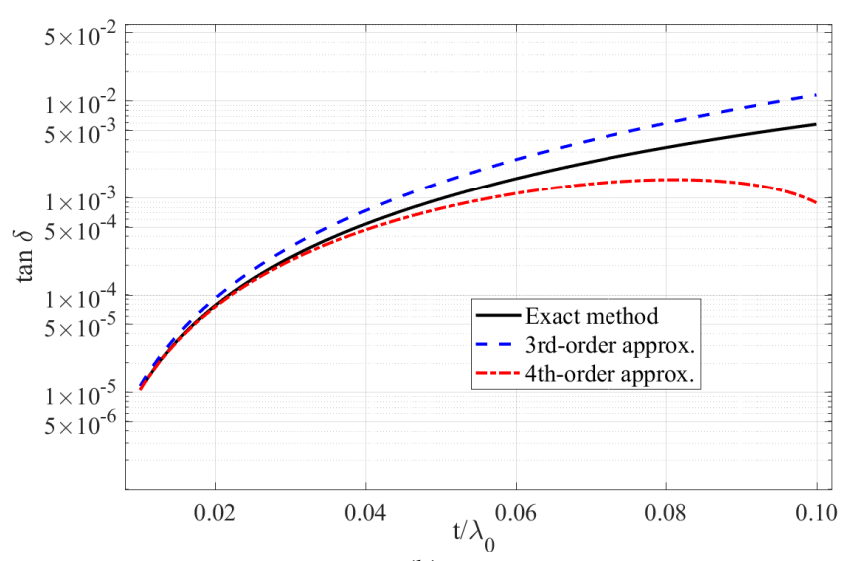

(b)

Fig. 3. Effective loss tangent as a function of the effective thickness $t / \lambda_{0}$ of the supporting side walls. (a) $L S E_{10}$ mode (propagating). (b) $L S E_{20}$ mode (in cut-off). The figure was produced for a waveguide with MDF supporting walls and a width $w=0.65 \lambda_{0}$.

$L S E_{10}$-mode and the first higher-order $L S E_{20}$-mode, which is in cut-off, are considered. A good agreement is observed for both modes. Since the third-order term is always positive and the fourth-order term always negative, the curve corresponding to the exact method is located between both approximations. Moreover, the approximation up to the third-order provides a worst-case estimate for the exact value of the effective loss tangent. Therefore, this approximation is applied to fix the optimal relative thickness ratio $t / w$ that yields acceptably low dielectric losses and substrate-independent behavior, while still guaranteeing sufficient mechanical stability. To support design choices, Table I shows the third-order approximation of the effective dielectric contrast and effective loss tangent of an AFSIW waveguide for different relative effective thicknesses $t / w$ of the supporting side walls, with the latter implemented in MDF, PMMA or PLA material. Only the fundamental mode is considered, but a similar table could be constructed for the higher-order modes. These modes remain in cut-off in the designs considered in this paper, but they may be exploited in more advanced multi-mode designs based on our new topology.

First, by fixing the relative thickness ratio to $t / w=0.05$, we ensure that the loss tangent remains below $10^{-4}$ while the dielectric permittivity maximally increases by about 0.005 compared to that of free space, for all materials under study. By applying (4) and making abstraction of the effect caused (a)

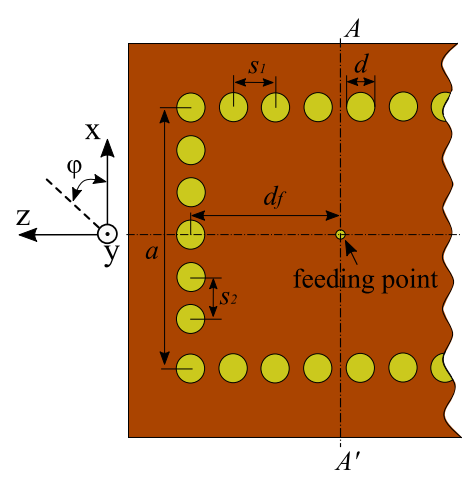

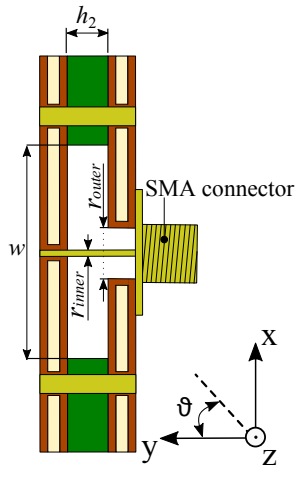

(b)
Fig. 4. Coaxial-to-air-filled SIW transition. (a) Top view. (b) Cross-sectional view at AA'. Optimized dimensions for all materials under study: $a=40.0 \mathrm{~mm}$, $w=36.0 \mathrm{~mm}, h_{2}=3.0 \mathrm{~mm}, d_{f}=31.0 \mathrm{~mm}, s_{1}=6.5 \mathrm{~mm}, s_{2}=6.78 \mathrm{~mm}$, $d=4.0 \mathrm{~mm}, r_{\text {outer }}=4.3 \mathrm{~mm}$ and $r_{\text {inner }}=1.3 \mathrm{~mm}$.

by the via walls, this corresponds to a width ratio $w / a$ in the order of 0.9. Second, based on the guidelines outlined in Section III-B and the set of available tubelets, we select the diameter of the vias to be $d=4.0 \mathrm{~mm}$. The relative via spacing is set to $s / d=1.625$. Substituting all values in (4) to account for the effect of the vias, yields $t / w=0.015$ as an improved estimate for the relative effective thickness for a width ratio $w / a=0.9$. Table I confirms that, for these design values, more than satisfactory performance is obtained.

\section{B. Coaxial-to-Air-Filled SIW Transition}

To implement AFSIW systems in smart surface applications with optimal performance, a carefully designed feed structure is needed. This transition should guarantee low insertion loss over a wide frequency band to meet the demanding requirements of state-of-the-art multimedia applications in terms of data rate and capacity. Moreover, its performance must remain independent of the dielectric properties of the off-the-shelf material serving as integration platform.

In [35], an open-ended coaxial-to-AFSIW transition was designed to yield a compact footprint and wideband behavior. However, our application scenario prefers a short-ended coaxial-to-AFSIW transition to minimize the radiation losses and to guarantee robust integration when installed in an actual smart surface system. This novel short-ended coaxial-to-AFSIW transition topology is shown in Fig. 4. In the transition part, brass tubelets serve as a metallic fence to confine the fields, as shown in Fig. 4. The coaxial probe feed is placed at a distance $d_{f}$ from the beginning of the waveguide. Its inner conductor is soldered to the upper conductor surface of the Layer I, while the outer conductor of the coaxial probe is connected to the bottom copper surface of Layer III.

The diameter of the hole in Layer III corresponds to the diameter of the outer conductor of the SMA connector. To eliminate parallel plate modes between lower and upper conductor layers of Layer III, the cylindrical via was plated during the PCB manufacturing process, as shown in Fig. 4(b). The same process is also applied between the lower and upper conductor layers of Layer I. The height and width of the AFSIW is fixed to $h_{2}=3.0 \mathrm{~mm}$ and $a=40 \mathrm{~mm}$, respectively, to ensure monomodal propagation within the desired frequency band, 


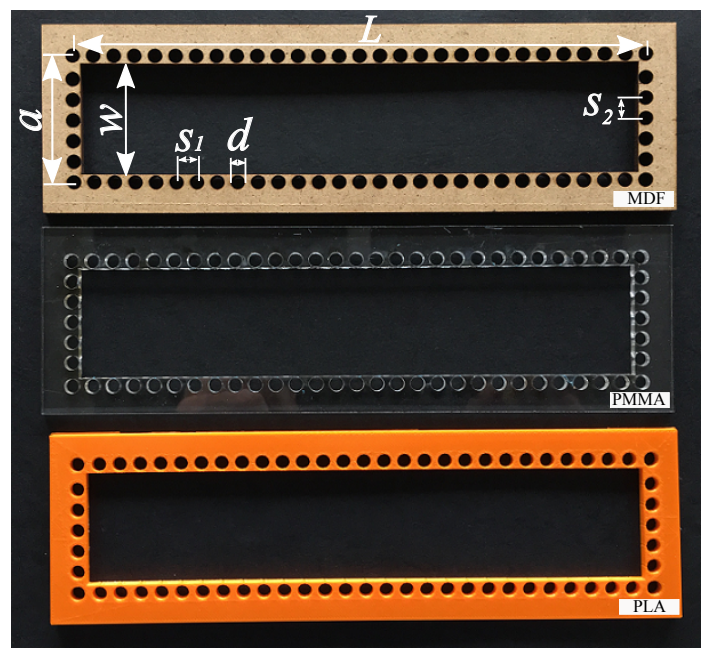

Fig. 5. Prototypes of Layer II of the [5.15-5.85] GHz AFSIW interconnection before assembly of Layers I-III. Optimized dimensions for all materials under study: $a=40.0 \mathrm{~mm}, w=36.0 \mathrm{~mm}, L=182.0 \mathrm{~mm}, s_{1}=6.5 \mathrm{~mm}$, $s_{2}=6.78 \mathrm{~mm}$, and $d=4.0 \mathrm{~mm}$. The thickness of Layer II is fixed to $h_{2}=3.0 \mathrm{~mm}$.

where only the dominant mode is guided by the waveguide. Computer-aided design using CST Microwave Studio yields the optimal dimensions of the transition, specified in the caption of Fig. 4. Note that these dimensions are independent of the material under consideration, owing to the procedure outlined in IV-A. Hence, our design exhibits substrate-independent performance in contrast to the dielectric-filled-to-air-filled SIW transition in [14]. This is now verified by evaluating identical components implemented in MDF, PMMA and PLA material.

\section{Air-Filled SIW Interconnections}

First, an AFSIW interconnection operating in the entire frequency band from $5.15 \mathrm{GHz}$ to $5.85 \mathrm{GHz}$ band is designed and fabricated. It consists of two coaxial-to-air-filled SIW transitions, as shown in Fig. 4, and an AFSIW waveguide of width $a=40 \mathrm{~mm}$ and a width ratio of $w / a=0.9$ in between. The latter has been selected according to the procedure outlined in Section IV-A to ensure monomodal propagation, while guaranteeing an effective dielectric permittivity and loss tangent below 1.005 and $10^{-4}$, respectively. As a result, an AFSIW interconnection of length $L=182.0 \mathrm{~mm}$ is realised to cover a distance of $120.0 \mathrm{~mm}$ between both ports. During the design procedure, the full-wave frequency-domain solver of CST Microwave Studio is used. Fig. 5 depicts three prototypes (one for each material under consideration) of Layer II of this AFSIW waveguide, before assembly of all layers. The MDF

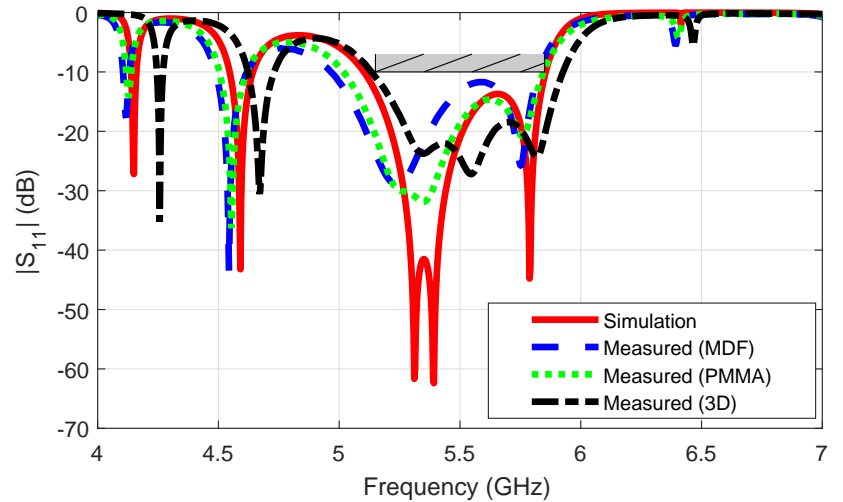

(a)

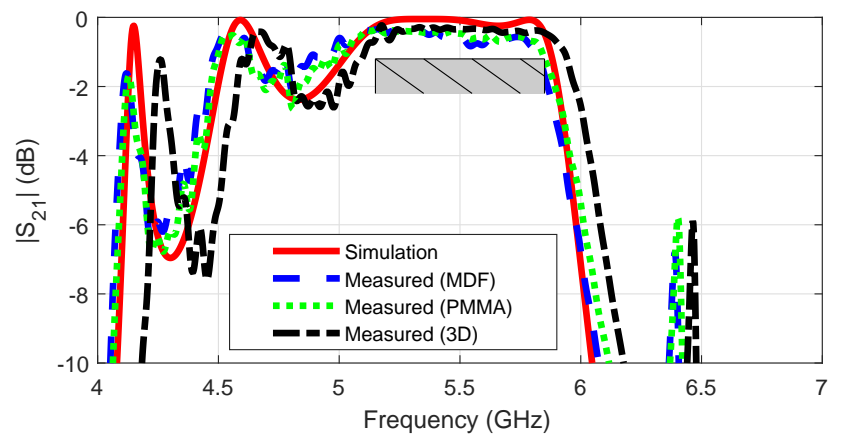

(b)

Fig. 6. S-parameters of AFSIW interconnections implemented in commerciallyavailable everyday surface materials.

and PMMA prototypes have been fabricated by standard CNC laser cutting (Section II.B), whereas the PLA prototype has been implemented through standard 3D printing (Section II.C). Layer I and Layer III are realized in a 0.5-mm-thick low-cost FR4 substrate $\left(\epsilon_{r}=4.5, \tan \delta=0.02\right)$, which is sufficiently thick for mechanical stability, this is, to prevent bending of these layers. An Electroless Nickel Immersion Gold (ENIG) surface finish was applied on all conductive layers on Layer I and Layer III. All layers were joined by brass tubelets with a diameter of $d=4.0 \mathrm{~mm}$.

Fig. 6 depicts the simulated and measured S-parameters of the three realized prototypes. Owing to the procedure to guarantee material-independent performance (Section IV-A), simulations are only performed for one material as support material. Specifically, Fig. 6 shows the simulated S-parameters for an AFSIW waveguide with sidewalls (Layer II) implemented in MDF. All measured results are in close agreement with these simulations, thereby demonstrating material-independent performance. As predicted by simulations, the measured $\left|S_{11}\right|$

TABLE I

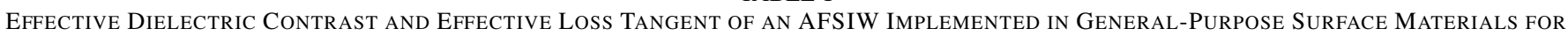
DifFerent Relative EFFeCtive Thicknesses $t / w$ OF the Supporting Side Walls.

\begin{tabular}{cc||ccc||ccc}
\multirow{2}{*}{$t / w$} & $\frac{4 \pi^{2}}{3}\left(\frac{t}{w}\right)^{3}$ & \multicolumn{3}{c||}{$\Delta \epsilon_{r, \text { eff }}$} & \multicolumn{3}{c}{$\tan \delta_{\text {eff }}$} \\
\hline & & MDF & PMMA & PLA (100\% Infill) & MDF & PMMA & PLA (100\% Infill) \\
\hline 0.010 & $1.32 \mathrm{e}-5$ & $1.6 \mathrm{e}-5$ & $2.7 \mathrm{e}-5$ & $2 \mathrm{e}-5$ & $7.9 \mathrm{e}-7$ & $6.6 \mathrm{e}-7$ & $1.2 \mathrm{e}-7$ \\
0.025 & $2.06 \mathrm{e}-4$ & $2.5 \mathrm{e}-4$ & $4.2 \mathrm{e}-4$ & $3.1 \mathrm{e}-4$ & $1.2 \mathrm{e}-5$ & $1.0 \mathrm{e}-5$ & $1.9 \mathrm{e}-6$ \\
0.050 & 0.0016 & 0.0020 & 0.0034 & 0.0025 & $9.9 \mathrm{e}-5$ & $8.2 \mathrm{e}-5$ & $1.5 \mathrm{e}-5$ \\
0.075 & 0.0056 & 0.0067 & 0.011 & 0.0083 & $3.3 \mathrm{e}-4$ & $2.8 \mathrm{e}-4$ & $5.2 \mathrm{e}-5$ \\
0.100 & 0.013 & 0.016 & 0.027 & 0.020 & $7.9 \mathrm{e}-4$ & $6.6 \mathrm{e}-4$ & $1.2 \mathrm{e}-4$ \\
0.125 & 0.026 & 0.031 & 0.053 & 0.039 & 0.0015 & 0.0013 & $2.4 \mathrm{e}-4$
\end{tabular}




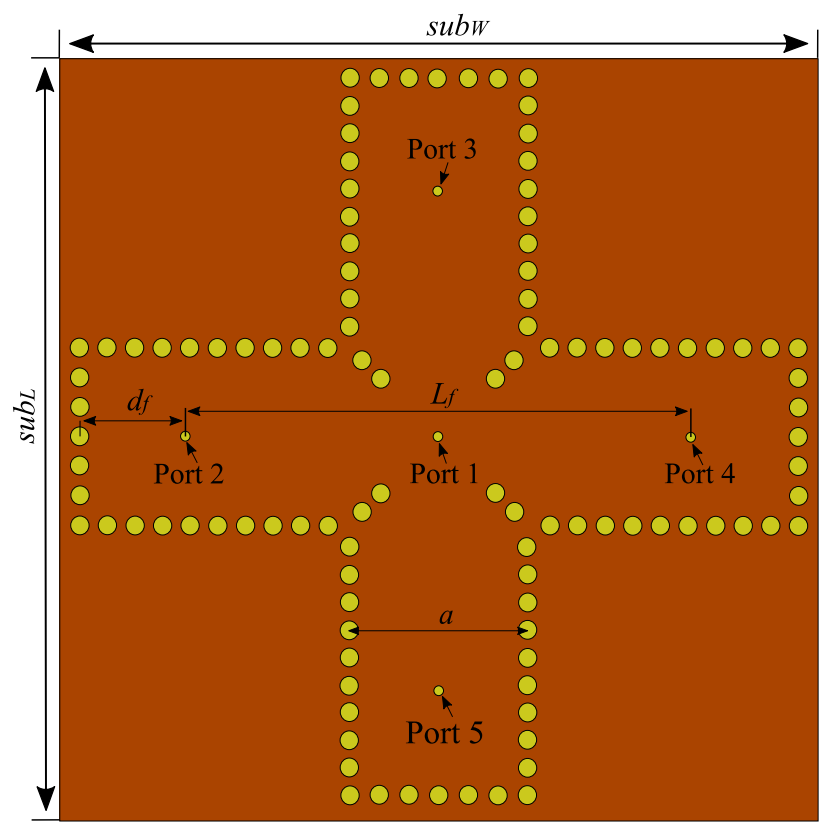

(a)

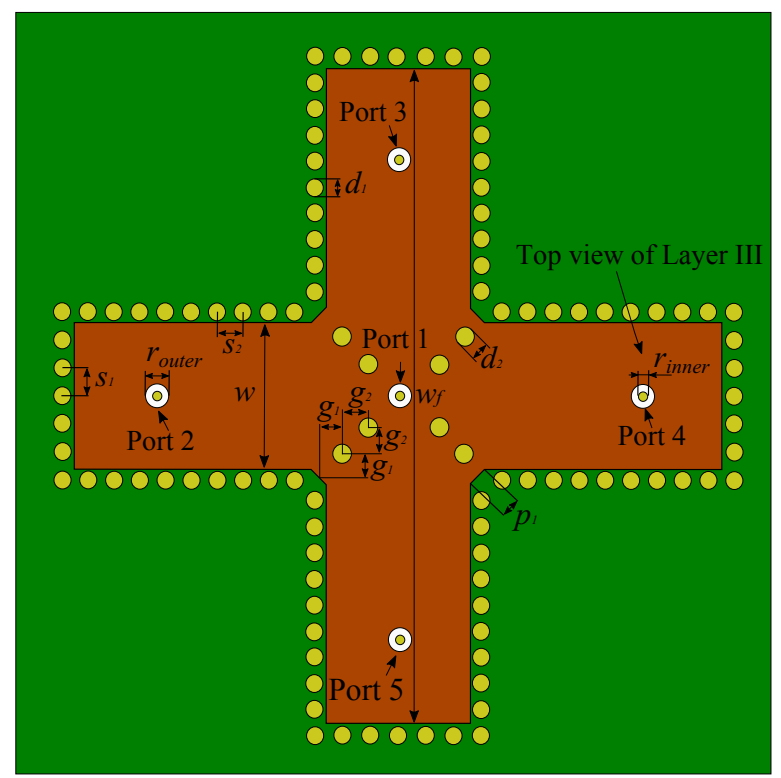

(b)

Fig. 7. Geometry of the proposed four-way power divider/combiner. (a) Top and bottom view of Layer I. (b) Cross-sectional view at Layer II. Optimized dimensions: $s u b_{L}=s u b_{W}=200.0 \mathrm{~mm}, h_{1}=h_{3}=0.5 \mathrm{~mm}, h_{2}=3.0 \mathrm{~mm}$, $w=39.15 \mathrm{~mm}, a=45.0 \mathrm{~mm}, L_{f}=130.6 \mathrm{~mm}, w_{f}=175.5 \mathrm{~mm}, d_{f}=25.7$ $\mathrm{mm}, s_{1}=7.5 \mathrm{~mm}, s_{2}=7.0 \mathrm{~mm}, p=5.66 \mathrm{~mm}, d_{1}=d_{2}=4.0 \mathrm{~mm}, g_{1}=2.78$ $\mathrm{mm}, g_{2}=4.6 \mathrm{~mm}, r_{\text {outer }}=4.3 \mathrm{~mm}$ and $r_{\text {inner }}=1.3 \mathrm{~mm}$.

[Fig. 6(a)] remains below $-10 \mathrm{~dB}$ over the entire frequency band of interest for all materials under study, indicating good matching with respect to $50 \Omega$. Furthermore, the measured insertion loss, including connector losses, remains below $1.5 \mathrm{~dB}$ over the entire frequency band from $5.15 \mathrm{GHz}$ to $5.85 \mathrm{GHz}$ for all prototypes. In particular, the measured insertion loss varies between $0.23 \mathrm{~dB}$ and $0.65 \mathrm{~dB}$ for the 3D-printed PLA prototype, between $0.32 \mathrm{~dB}$ and $1.2 \mathrm{~dB}$ for the CNC-laser-cut PMMA prototype, and between $0.32 \mathrm{~dB}$ and $1.5 \mathrm{~dB}$ for the CNC-laser-cut MDF prototype. However, the slightly higher worst-case insertion loss for the PMMA (1.2 dB) and MDF

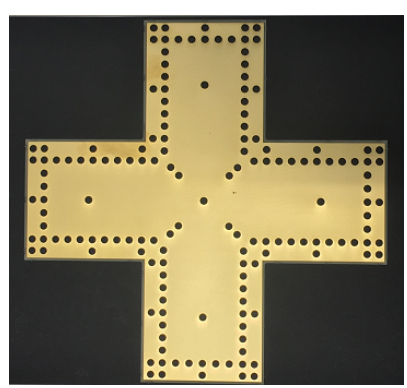

(a)

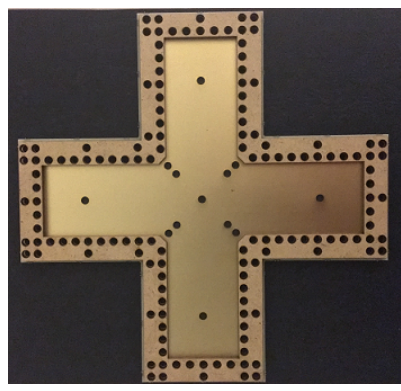

(c)

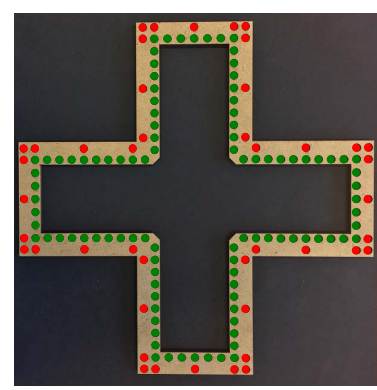

(b)

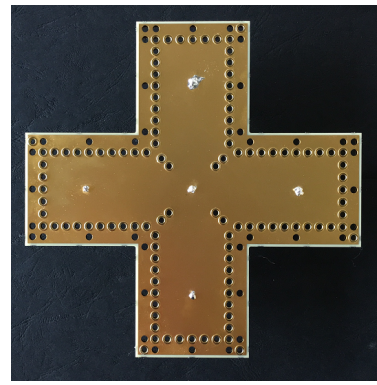

(d)
Fig. 8. Prototype of the stand-alone four-way power divider/combiner with MDF sidewalls (Layer II). (a) Top view of Layer III. (b) Top view of Layer II [red holes for tubelets to ensure mechanical stability and green holes for AFSIW sidewall tubelets]. (c) Layer II aligned with Layer III. (d) Assembled Prototype.

$(1.5 \mathrm{~dB})$ prototypes can be attributed to fabrication inaccuracies, causing a small shift of the measured $\left|S_{21}\right|$ curve to lower frequencies. In fact, the measured insertion loss remains below $0.85 \mathrm{~dB}$ from $5.0 \mathrm{GHz}$ to $5.80 \mathrm{GHz}$, for the MDF prototype, and from $5.05 \mathrm{GHz}$ to $5.83 \mathrm{GHz}$, for the PMMA prototype. Hence, we conclude that our novel AFSIW manufacturing technology allows the implementation of high-performance microwave components inside a wide variety of traditional everyday surface materials by standard additive or subtractive manufacturing processes.

\section{Power Divider}

1) Design: We now exploit our novel AFSIW implementation technology to develop a more complex-shaped microwave component, being a four-way power divider/combiner. In addition, we demonstrate robust and invisible integration inside a worktop of a desk. Such a component may be used to distribute wideband signals in the next generation smart desk applications that exploit multi-antenna and/or multi-transmitter/receiver systems. Fig. 7 depicts the proposed geometry. When used as a power divider, the SMA connector in the center of the structure (Port 1) acts as input. The signal injected into this port is equally divided over the other four SMA outputs (Ports 2 to 5), via a coaxial-to-air-filled SIW transition. When serving as a power combiner, the signals injected into the latter four SMA connectors (Port 2 to 5) will constructively sum up at the center SMA output (Port 1). Eight inductive posts (tubelets) are introduced near Port 1 to minimize transmission loss and reflection at the input ports, while maximizing isolation between Ports 2 to 5. Based on the guidelines provided in Section IV-A and the results obtained in Section IV-C, initial dimensions 


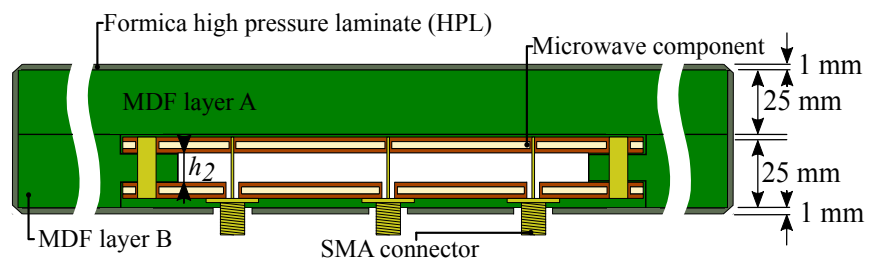

(a)

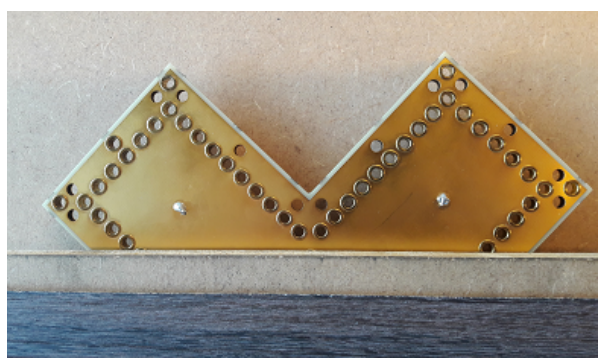

(b)

Fig. 9. Integration of the four-way power/divider inside a worktop. (a) Crosssectional view. (b) Prototype before thermally bonding the HPL layers.

were proposed to minimize dielectric losses and to guarantee material-independent performance. Next, computer-aided fullwave optimization using CST Microwave Studio yields the optimal dimensions, shown in the caption of Fig. 7.

2) Fabrication and Integration: First, a stand-alone four-way power divider/combiner is implemented in MDF through lowcost CNC laser-cutting, following Section II-B, and assembly, according to Section II-D. Fig. 8 shows the assembly process and the final stand-alone prototype. As in Section IV-C, a 0.5mm-thick low-cost FR4 PCB substrate is used to implement Layer I and Layer III [Fig. 8(a)], after which brass tubelets with a diameter of $d_{1}=d_{2}=4.0 \mathrm{~mm}$ are applied to align and assemble all layers. Similar tubelets were used to implement the inductive posts. By carefully setting the hand eyelet press, no additional dielectric slabs were needed to support these tubelets. Furthermore, additional tubelets may be implemented near the AFSIW sidewalls, as shown in Fig. 8(b), to further enhance mechanical stability.

Next, the proposed four-way power divider is invisibly integrated inside the worktop of a desk. The selected worktop consists of two 25-mm-thick MDF layers, sandwiched between two layers of 1-mm-thick Formica high-pressure laminate (HPL). This material stack-up was selected as an integration platform, as such panels are commonly used to manufacture worktops because of their high wear resistance, durability, and processing ability. The integration process is quite similar to the fabrication process of the stand-alone prototype. It is depicted in Fig. 9. First, the exact shape of the air-filled regions of the power divider/combiner is removed by standard $\mathrm{CNC}$ lasercutting in MDF layer B [Fig. 9(a)], according to the geometry in Fig. 7. Next, a recess is milled out in the same MDF layer to accommodate for Layer I and Layer III. Extra care is taken to ensure a thickness $h_{2}=3.0 \mathrm{~mm}$ between Layers I and III. Finally, MDF layer A and both HPL layers are thermally bonded to MDF layer B, as shown in Fig. 9(a). Fig. 9(b) depicts the integrated prototype just before assembling all layers. Consequently, this results in a robust and invisible

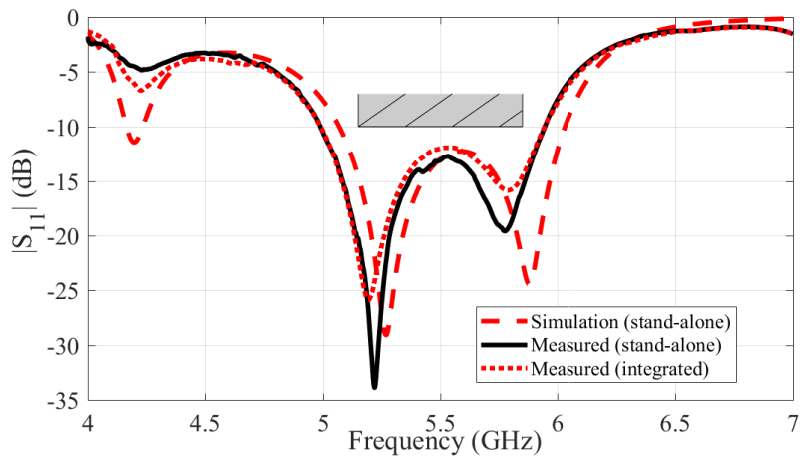

(a)

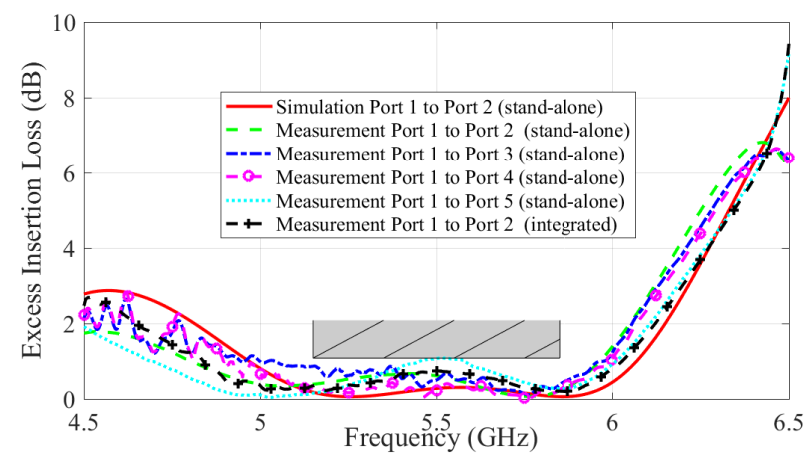

(b)

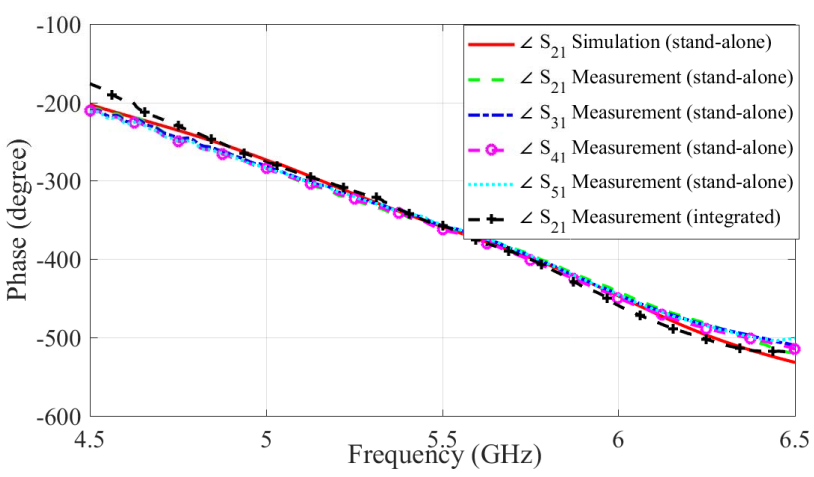

(c)

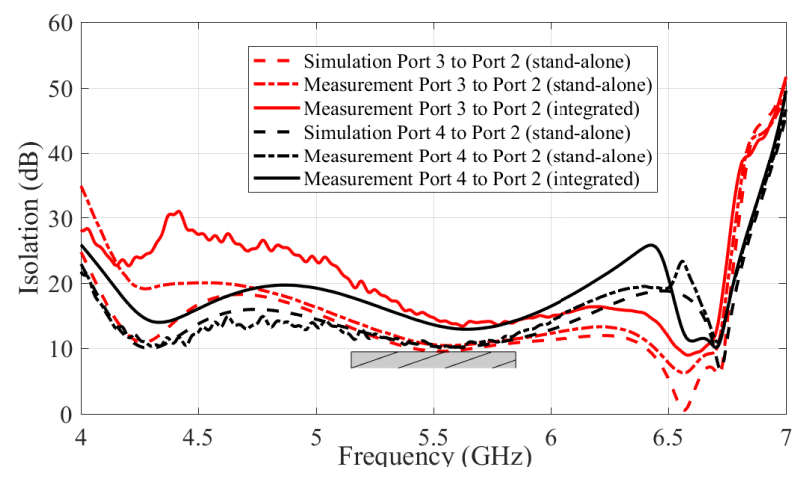

(d)

Fig. 10. Measurement and simulation results of the AFSIW power divider. (a) Reflection coefficient of input port. (b) Insertion loss in excess of the $6 \mathrm{~dB}$ coupling loss. (c) Phase balance between output ports. (d) Isolation between output ports. 
integration into a common surface.

3) Measurement: First, the stand-alone performance of the air-filled SIW four-way power divider/combiner was validated by measuring its S-parameters with an Agilent N5242A PNA-X Microwave Network Analyzer. Fig. 10(a) shows a good agreement between measured and simulated reflection coefficients at Port 1 , demonstrating good impedance matching in the complete frequency band from $5.15 \mathrm{GHz}$ to $5.85 \mathrm{GHz}$. Fig. 10(b) showcases good agreement between measured and simulated power insertion loss, in excess of the $6 \mathrm{~dB}$ coupling loss. The measured excess insertion loss and amplitude imbalance between the four branches remain below $1 \mathrm{~dB}$ over the entire frequency band of interest. Furthermore, Fig. 10(c) depicts the phase behaviour of each of the four branches, showing good symmetry and a maximum phase imbalance of $7^{\circ}$ within the band of interest. Finally, Fig. 10(d) demonstrates that the isolation between the four output ports remains above $10 \mathrm{~dB}$ in the entire frequency band of operation. Moreover, the performance of the four-way power divider was validated after integration inside a worktop according to Fig. 9. The results are also depicted in Fig. 10. The excellent shielding of the AFSIW topology ensures that the integration procedure only has marginal influence on its performance. Based on these measurements, we conclude that our novel approach enables direct integration of a wide range of high-performance microwave components into everyday surfaces, without altering their appearance or original purpose. Hence, the way is paved for a new family of cost-effective, highperformance and invisibly-integrated smart surface systems that exploit the area and the materials available in everyday objects.

\section{CONCLUSION}

An innovative architecture was proposed to realize highperformance microwave components in a substrate- and manufacturing-process-independent low-loss SIW implementation technology. Therefore, general-purpose commerciallyavailable surface materials were judiciously shaped such that the strongest electromagnetic fields occur in air-filled regions, while low-cost tubelets further minimize fields in these lossy materials. An analytical approximation was derived for the effective dielectric contrast and loss tangent of such an AFSIW waveguide, as a function of the thickness and the electromagnetic properties of the dielectric slabs supporting the sidewalls. This formula was leveraged in the design process to guarantee low losses and material-independent performance. As a result, any off-the-shelf surface material processed through standard subtractive (CNC milling/lasercutting) or additive (3Dprinting) manufacturing processes can be applied as a substrate for such microwave components. This yields a complete substrate-independent high-performance microwave system that is directly and invisibly integrated into traditional everyday surface materials, turning them into smart surfaces in a costeffective way. As a proof of concept, a coaxial-to-air-filled SIW transition, a waveguide and a power divider were fabricated and integrated into different commonly-used surface materials. Measurements prove that all prototypes exhibit excellent performance within the entire frequency band from $5.15 \mathrm{GHz}$ to $5.85 \mathrm{GHz}$ band. Hence, the designed components are very suitable for seamless integration into everyday surfaces.
Finally, our novel manufacturing technology also opens up new perspectives for future research. First, even lower losses may be pursued in future designs by exploiting multiple rows of vias, of which one or more rows ensure mechanical stability and one row defines AFSIW structures in a completely air-filled region. Second, the 3D-printing technology may be leveraged to realize more complex-shaped non-planar AFSIW microwave components, thereby paving the way for cost-effective curved smart surface systems. Third, to achieve operation over an even broader bandwidth, novel feeding topologies may be developed and/or combined with multi-moding bandwidth enhancement techniques.

\section{REFERENCES}

[1] G. Kortuem, F. Kawsar, V. Sundramoorthy, and D. Fitton, "Smart objects as building blocks for the Internet of Things," IEEE Internet Comput., vol. 14, no. 1, pp. 44-51, Feb. 2010.

[2] H. Sundmaeker, P. Guillemin, P. Friess, and S. Woelffle, "Vision and challenges for realising the Internet of Things," European Commision Cluster of European Research Projects on the Internet of Things, vol. 3, no. 3, pp. 34-36, 2010.

[3] L. Roselli et al., "Smart surfaces: Large area electronics systems for Internet of Things enabled by energy harvesting," Proc. IEEE, vol. 102, no. 11, pp. 1723-1746, Nov. 2014.

[4] R. Moro, S. Agneessens, H. Rogier, A. Dierck, and M. Bozzi, "Textile microwave components in substrate integrated waveguide technology," IEEE Trans. Microw. Theory Techn., vol. 63, no. 2, pp. 422-432, Feb. 2015.

[5] M. Ito et al., "Smart furniture: improvising ubiquitous hot-spot environment," in Int. Conf. on Distrib. Comput. Syst. Workshops, Providence, Rhode Island, USA, 2003, pp. 248-253.

[6] O. Caytan et al., "Half-mode substrate-integrated-waveguide cavitybacked slot antenna on cork substrate," IEEE Antennas Wireless Propag. Lett., vol. 15, pp. 162-165, May 2016.

[7] S. Lemey et al., "Threefold rotationally symmetric SIW antenna array for ultra-short-range MIMO communication," IEEE Trans. Antennas Propag., vol. 64, no. 5, pp. $1689-1699$, May 2016.

[8] T. Castel, S. Lemey, P. Van Torre, C. Oestges, and H. Rogier, "Fourelement ultrawideband textile cross array for dual-spatial and dualpolarization diversity," IEEE Antennas Wireless Propag. Lett., vol. 16, pp. 481-484, Jun. 2017.

[9] M. Bozzi, A. Georgiadis, and K. Wu, "Review of substrate-integrated waveguide circuits and antennas," IET Microw. Antennas Propag., vol. 5, no. 8, pp. 909-920, 2011.

[10] M. Esquius-Morote, B. Fuchs, J.-F. Zurcher, and J. R. Mosig, "Novel thin and compact H-plane SIW horn antenna," IEEE Trans. Antennas Propag., vol. 61, no. 6, pp. 2911-2920, Jun. 2013.

[11] M. Bozzi et al., "Novel materials and fabrication technologies for SIW components for the Internet of Things," in IEEE Int. Workshop on Electromagn.: Appl. and Student Innovation Competition (iWEM), Nanjing, China, 2016, pp. 1-3.

[12] S. Lemey, O. Caytan, D. V. Ginste, P. Demeester, H. Rogier, and M. Bozzi, "SIW cavity-backed slot (multi-) antenna systems for the next generation IoT applications," in IEEE Topical Conf. on Wireless Sensors and Sensor Netw. (WiSNet), Austin, TX, USA, 2016, pp. 75-77.

[13] N. Ranjkesh and M. Shahabadi, "Reduction of dielectric losses in substrate integrated waveguide," Electron. Lett., vol. 42, no. 21, pp. 1230-1232, Oct. 2006

[14] F. Parment, A. Ghiotto, T.-P. Vuong, J.-M. Duchamp, and K. Wu, “Airfilled substrate integrated waveguide for low-loss and high power-handling millimeter-wave substrate integrated circuits," IEEE Trans. Microw. Theory Techn., vol. 63, no. 4, pp. 1228-1238, Apr. 2015.

[15] — , "Low-loss air-filled substrate integrated waveguide (SIW) band-pass filter with inductive posts," in Eur. Microw. Conf. (EuMC), Paris, France, 2015, pp. 761-764.

[16] , "Double dielectric slab-loaded air-filled SIW phase shifters for highperformance millimeter-wave integration," IEEE Trans. Microw. Theory Techn., vol. 64, no. 9, pp. 2833-2842, Sep. 2016.

[17] J. Digby et al., "Fabrication and characterization of micromachined rectangular waveguide components for use at millimeter-wave and terahertz frequencies," IEEE Trans. Microw. Theory Techn., vol. 48, no. 8, pp. 1293-1302, Aug. 2000. 
[18] A. Isapour and A. B. Kouki, "Empty LTCC integrated waveguide with compact transitions for ultra-low loss millimeter-wave applications," IEEE Microw. Compon. Lett., vol. 27, no. 2, pp. 144-146, Feb. 2017.

[19] P. Mohammadi and S. Demir, "Loss reduction in substrate integrated waveguide structures," Progress In Electromagn. Res. C, vol. 46, pp. 125133, 2014.

[20] F. Bigelli et al., "Design and fabrication of a dielectricless substrateintegrated waveguide," IEEE Trans. Compon. Packag. Manuf. Technol., vol. 6, no. 2, pp. 256-261, Feb. 2016.

[21] A. Belenguer, J. L. Cano, H. Esteban, E. Artal, and V. E. Boria, "Empty substrate integrated waveguide technology for E-plane high-frequency and high-performance circuits," Radio Sci., vol. 52, no. 1, pp. 49-69, Jan. 2017.

[22] Q. Van den Brande, S. Lemey, J. Vanfleteren, and H. Rogier, "High efficient impulse-radio ultra-wideband cavity backed slot antenna in stacked air-filled substrate-integrated-waveguide technology," IEEE Trans. Antennas Propag., submitted.

[23] A. Belenguer, H. Esteban, and V. E. Boria, "Novel empty substrate integrated waveguide for high-performance microwave integrated circuits," IEEE Trans. Microw. Theory Techn., vol. 62, no. 4, pp. 832-839, Apr 2014.

[24] M. Rossi, S. Agneessens, H. Rogier, and D. V. Ginste, "Assembly-linecompatible electromagnetic characterization of wearable antenna substrates," IEEE Antennas Wireless Propag. Lett., vol. 16, pp. 1365-1368, Dec. 2016.

[25] W. J. Otter and S. Lucyszyn, "3-D printing of microwave components for 21 st century applications," in IEEE Int. Workshop on Electromagnetics: Appl. and Student Innovation Competition (iWEM), Chengdu, China, 2016, pp. 1-3.

[26] D.-K. Ahn, S.-M. Kwon, and S.-H. Lee, "Expression for surface roughness distribution of FDM processed parts," in Int. Conf. on Smart Manuf. Appl., Gyeonggdi-do, South-Korea, 2008, pp. 490-493

[27] S. Moscato et al., "Additive manufacturing of 3D substrate integrated waveguide components," Electron. Lett., vol. 51, no. 18, pp. 1426-1428, Aug. 2015.

[28] S. Lemey, F. Declercq, and H. Rogier, "Textile antennas as hybrid energyharvesting platforms," Proc. IEEE, vol. 102, no. 11, pp. 1833-1857, Nov. 2014.

[29] R. E. Collin, Inhomogeneously filled waveguides and dielectric resonators, 1st ed. Wiley-IEEE Press, 1991, ch. 6, pp. 411-470.

[30] Z. Kordiboroujeni and J. Bornemann, "Designing the width of substrate integrated waveguide structures," IEEE Microw. Compon. Lett., vol. 23, no. 10 , pp. 518-520, Oct. 2013.

[31] M. Bozzi, L. Perregrini, and K. Wu, "Modeling of radiation, conductor, and dielectric losses in SIW components by the BI-RME method," in Eur. Microw. Integr. Circuit Conf., Amsterdam, The Netherlands, 2008 , pp. 230-233.

[32] W. Ayres, P. Vartanian, and A. Helgesson, "Propagation in dielectric slab loaded rectangular waveguide," IEEE Trans. Microw. Theory Techn., vol. 6, no. 2, pp. 215-222, Apr. 1958.

[33] F. Declercq, H. Rogier, and C. Hertleer, "Permittivity and loss tangent characterization for garment antennas based on a new matrix-pencil twoline method," IEEE Trans. Antennas Propag., vol. 56, no. 8, pp. 25482554, Aug. 2008.

[34] S. Moscato et al., "Infill-dependent 3-D-printed material based on NinjaFlex filament for antenna applications," IEEE Antennas Wireless Propag. Lett., vol. 15, pp. 1506-1509, Jan. 2016.

[35] A. A. Khan and M. K. Mandal, "A compact broadband direct coaxial line to SIW transition," IEEE Microw. Compon. Lett., vol. 26, no. 11, pp. 894-896, Nov. 2016

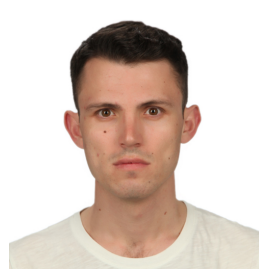

Kamil Yavuz Kapusuz (S'17) was born in 1988. He received the M.Sc. degree in electrical engineering from Atilim University, Ankara, Turkey in 2013. He is currently pursuing the Ph.D. degree in electrical engineering with the Electromagnetics Group, Department of Information Technology, Ghent, Belgium.

From 2014 to 2016, he was with Remote Sensing Technologies, Ankara, Turkey. He developed antenna arrays at millimeter-wave frequencies for radar applications. He also developed scalable phased array antennas for SATCOM-on-the-move concepts. His research interests include design and development of passive RF components, multifunction antennas, reconfigurable antennas, and phased arrays.

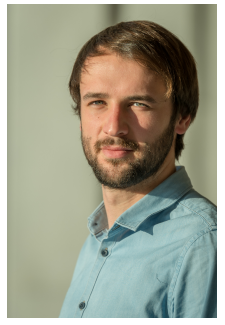

Sam Lemey (S'14 - M'16) received the M.Sc. degree in electronic engineering from Howest, University College West Flanders, Kortrijk, Belgium, in 2012 and the Ph.D. degree in electrical engineering from Ghent University, Ghent, Belgium, in 2016.

$\mathrm{He}$ is currently working as a Post-Doctoral researcher at the Electromagnetics Group in the Department of Information Technology at Ghent University. His research focuses on robust antenna systems for wearable applications, energy-harvesting techniques for wireless nodes, active antenna design for the Internet of Things and 5G applications, IR-UWB antenna systems for centimeterprecision localization, novel techniques to implement substrate integrated waveguide structures in unconventional materials, and full-wave/circuit cooptimization frameworks to realize active antenna systems.

Dr. Lemey received the Best Paper Award at the 2016 IEEE MTT-S Topical Conference on Wireless Sensors and Sensor Networks.

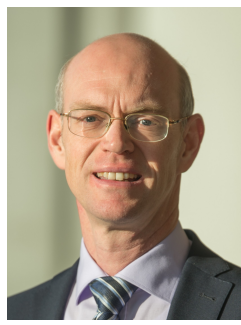

Hendrik Rogier (SM'06) was born in 1971 in Geraardsbergen. He received the M.Sc. and Ph.D. degrees in electrical engineering from Ghent University, Ghent, Belgium, in 1994 and 1999, respectively.

From 2003 to 2004, he was a Visiting Scientist with the Mobile Communications Group, Vienna University of Technology, Vienna, Austria. He is a currently a Full Professor with the Department of Information Technology, Ghent University, a Guest Professor with the Interuniversity Microelectronics Centre, Ghent, and a Visiting Professor with the University of Buckingham, Buckingham, U.K. He has authored or co-authored over 145 papers in international journals and over 160 contributions in conference proceedings. His current research interests include antenna systems, radio wave propagation, body-centric communication, numerical electromagnetics, electromagnetic compatibility, and power/signal integrity.

Dr. Rogier is a member of Technical Committee 24 on RFID Technology with the IEEE Microwave Theory and Techniques Society (MTT-S) and a member of the Governing Board of Topical Group MAGEO on Microwaves in Agriculture, Environment and Earth Observation with the European Microwave Association, Leuven, Belgium. He was a recipient of the URSI Young Scientist Award (twice) at the 2001 URSI Symposium on Electromagnetic Theory and at the 2002 URSI General Assembly, the 2014 Premium Award for Best Paper in the IET Electronics Letters, the Best Paper Award First Place in the 2016 IEEE MTT-S Topical Conference on Wireless Sensors and Sensor Networks, the Best Poster Paper Award at the 2012 IEEE Electrical Design of Advanced Packaging and Systems Symposium, the Best Paper Award at the 2013 IEEE Workshop on Signal and Power Integrity, and the Joseph Morrissey Memorial Award for the First Best Scientific Paper at BioEM 2013. He is an Associate Editor of IET Electronics Letters, IET Microwaves, Antennas and Propagation, and the IEEE TRANSACTIONS ON MTT. He acts as the URSI Commission B representative for Belgium. 